\title{
Helping smokers with cardiac disease to abstain from tobacco after a stay in hospital
}

\author{
Nancy A. Rigotti MD
}

$\infty \quad$ See related research article by Smith and Burgess, page 1297

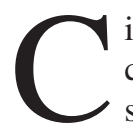
igarette smoking is a major risk factor for cardiovascular disease. Quitting smoking clearly benefits smokers, including those who quit even after cardiovascular disease has developed. In a meta-analysis of 20 studies, people who stopped smoking after a heart attack or cardiac surgery had a $36 \%$ risk reduction for subsequent cardiovascular mortality compared with those who did not stop smoking. ${ }^{1}$ These data illustrate why addressing the use of tobacco should be a cornerstone of the secondary prevention of cardiovascular disease. Unfortunately, smoking receives far less attention from cardiologists than other cardiovascular risk factors. ${ }^{2,3}$ In this issue, a study by Smith and Burgess ${ }^{4}$ provides further evidence that this should change.

This study builds on a classic study from 1990 by Taylor and colleagues ${ }^{5}$ at Stanford Medical School. Their randomized controlled trial demonstrated the efficacy of a nursebased counselling program for smoking cessation among patients admitted to hospital for myocardial infarction (MI) and who wanted to stop smoking. A trained nurse provided cognitive-behavioural counselling at the patient's bedside and made up to 6 scheduled telephone calls in the 4 months after discharge. The program nearly doubled the smoking cessation rate at 1 year after hospital discharge, from $32 \%$ in the usual care group to $61 \%$ in the intervention group.

This landmark study was followed by other trials that collectively established hospital-based interventions for smoking cessation as "best practice" for patients after MI. In a metaanalysis of 18 randomized controlled trials involving smokers admitted to hospital for cardiovascular disease, bedside counselling followed by more than 1 month of supportive contact after discharge, usually by telephone, was associated with an $81 \%$ increase in the odds of quitting compared with usual care. ${ }^{6}$

The counselling protocol in the study by Smith and Burgess consisted of 1 hour of nurse counselling in hospital followed by 7 telephone calls over 2 months after discharge. Their protocol resembled the one used in the original trial by Taylor and colleagues and achieved remarkably similar results. It is reassuring that the counselling intervention is still effective decades after the original trial, even though patients with MI now spend less time in hospital than in the 1980s. Presumably, the intervention is still effective because so much of it occurs after hospital discharge. The importance of the postdischarge component was highlighted by the findings of a recent meta-analysis of in-hospital interventions for smoking cessation. ${ }^{6}$ In this analysis, counselling that occurred only in

\section{Key points}

- Smoking cessation is a critical but often neglected component of the secondary prevention of cardiovascular disease.

- Smokers who are admitted to hospital for cardiovascular disease benefit from smoking-cessation counselling that begins in hospital and continues for more than 1 month after discharge.

- Interventions for smoking cessation initiated in hospital need to be translated from research into routine clinical practice.

the hospital without sustained contact after discharge did not improve long-term smoking cessation rates over usual care.

Despite the similarities between the trials, the new trial by Smith and Burgess differs from the original trial in several ways. First, Smith and Burgess showed that the program was effective not only for patients admitted because of MI but also for smokers who were admitted for coronary artery bypass graft surgery. Second, few patients in the original trial had used smoking-cessation medications, whereas one-third of the patients in the trial by Smith and Burgess used these medications, reflecting the greater use of and expanding options for pharmacotherapy over the intervening decades.

Paradoxically, smokers in both arms of the new trial who used pharmacotherapy had lower cessation rates than those who did not use such medications. This apparent contradiction has been observed in other studies in which smokers choose whether or not to use pharmacotherapy.? The finding can be explained if smokers who are less likely to succeed in quitting because of stronger nicotine dependence are the same smokers who choose to use cessation medication. ${ }^{7}$ In the trial by Smith and Burgess, smokers who used pharmacotherapy benefited more from the counselling intervention (an increase in the cessation rate from $22 \%$ to $55 \%$ ) than did smokers who used no medication (an increase in the cessation rate from $60 \%$ to $76 \%$ ). This interaction was not statistically significant, owing in part to the small sample size in the subgroup analysis. However, these results suggest that a combination of pharmacotherapy and counselling may offer hope to some smokers whose efforts otherwise stand a small chance of success.

Thanks to this and previous trials, strong evidence sup-

Nancy A. Rigotti directs the Tobacco Research and Treatment Center of the Massachusetts General Hospital and is Professor of Medicine at Harvard Medical School, Boston, USA. 
ports the efficacy of smoking interventions for inpatients with cardiovascular disease.

Still, challenges remain. Almost half of the smokers in the current study resumed smoking within 1 year, despite experiencing a serious tobacco-related health consequence, undergoing a period of enforced tobacco abstinence during their stay in hospital and receiving an intensive hospital-initiated intervention. The fact that so many patients resumed smoking is testimony to the addictive nature of tobacco and illustrates the need for more powerful interventions. One strategy would be to add pharmacotherapy to the comprehensive hospitalinitiated counselling model. The combination of medication and counselling is synergistic in outpatients and is the current standard of care. ${ }^{8}$ Whether this holds for smokers while in hospital should be explored.

Another challenge is translating the research evidence base into routine clinical practice. Krumholz and colleagues ${ }^{9}$ found that the counselling-based intervention used by Taylor and colleagues $^{5}$ was more cost-effective than other medical therapies considered to be standard care among patients with MI. More recently, a study that followed for 2 years patients with MI found that an intensive intervention reduced readmissions and all-cause mortality. ${ }^{10}$ The results of these studies suggest that, if widely adopted, interventions for smoking cessation have the potential to reduce cardiovascular morbidity and mortality at the population level. Unfortunately, almost 20 years after the original study, widespread implementation has not occurred. "Best practice" has not yet become "usual care."

A major problem is that the intervention model does not fit neatly into the existing delivery system for health care. Implementing the model requires coordinating care from the inpatient to the outpatient setting. Bridging this gap is a challenge for all chronic diseases. Addiction to tobacco is no exception. Hospitals do not see care after discharge as their responsibility and are not eager to bear the cost. Treatment for dependence on tobacco is not well covered by health insurers and payers, who are not eager to add new benefits in an era of soaring health care costs, even if the therapies are cost-effective.

In the United States, change was stimulated when hospital accrediting organizations adopted new quality-of-care standards in 2004. These organizations measured whether smok- ers with acute MI were given advice on smoking cessation or assistance during their hospital stay. The results are publicly reported and appear to have stimulated hospital-based counselling efforts. How well these new programs adhere to the evidence-based models that are needed to achieve long-term smoking cessation is uncertain, but it is a promising start. Sixteen years ago, Orleans and Ockene ${ }^{11}$ called routine hospitalbased smoking counselling for patients with MI "an idea whose time has come." It is well overdue.

Competing interests: Nancy Rigotti has received payment and travel expenses from Pfizer, which makes a smoking-cessation medication, and Free and Clear, which provides telephone-based counselling for smoking cessation. She has received research grants from Pfizer, Sanofi Aventis and Nabi Biopharmaceuticals for studies of approved and investigational smoking cessation products.

\section{REFERENCES}

1. Critchley JA, Capewell S. Mortality risk reduction associated with smoking cessation in patients with coronary heart disease: a systematic review. JAMA 2003;290: 86-97.

2. Erhardt L. Cigarette smoking: an undertreated risk factor for cardiovascular disease. Atherosclerosis DOI: 10.1016/j.atherosclerosis.2009.01.007. Epub 2009 Jan 15 ahead of print.

3. Cardiologists should be less passive about smoking cessation [editorial]. Lancet 2009;373:867

4. Smith P, Burgess ED. Smoking cessation initiated during hospital stay for patients with coronary artery disease: a randomized controlled trial. CMAJ 2009;180:12971303.

5. Taylor CB, Houston-Miller N, Killen JD, et al. Smoking cessation after acute myocardial infarction: effects of a nurse-managed intervention. Ann Intern Med 1990; 113:118-23.

6. Rigotti NA, Munafo MR, Stead LF. Smoking cessation interventions for hospitalized smokers: a systematic review. Arch Intern Med 2008;168:1950-60.

7. Shiffman S, Brockwell SE, Pillitteri JL, et al. Use of smoking-cessation treatments in the United States. Am J Prev Med 2008;34:102-11.

8. Fiore MC, Jaén CR, Baker TB, et al. Treating tobacco use and dependence: 2008 update. Clinical practice guidelines. Rockville (MD): US Department of Health and Human Services, Public Health Service; 2008

9. Krumholz HM, Cohen BJ, Tsevat J, et al. Cost-effectiveness of a smoking cessation program after myocardial infarction. J Am Coll Cardiol 1993;22:1697-702

10. Mohiuddin SM, Mooss AN, Hunter CB, et al. Intensive smoking cessation intervention reduces mortality in high-risk smokers with cardiovascular disease. Chest 2007;131:446-52.

11. Orleans CT, Ockene JK. Routine hospital-based quit-smoking treatment for the postmyocardial infarction patient: an idea whose time has come. J Am Coll Cardiol 1993;22:1703-5

Correspondence to: Dr. Nancy A. Rigotti, Tobacco Research and Treatment Center, Massachusetts General Hospital, 50 Staniford St., 9th fl., Boston, MA 02114,USA; nrigotti@partners.org 